\title{
Novel Porous Polymorphs of Zinc Cyanide with Rich Thermal and Mechanical Behavior
}

\author{
Fabien Trousselet, ${ }^{\dagger}$ Anne Boutin, ${ }^{* \dagger}{ }^{\dagger}$ and François-Xavier Coudert ${ }^{*} \neq$ \\ †École Normale Supérieure, PSL Research University, Département de Chimie, Sorbonne Universités - UPMC Univ Paris 06, CNRS \\ UMR 8640 PASTEUR, 24 rue Lhomond, 75005 Paris, France \\ ${ }^{\ddagger}$ PSL Research University, Chimie ParisTech - CNRS, Institut de Recherche de Chimie Paris, 75005 Paris, France
}

Supporting Information

ABSTRACT: We investigate the feasibility of four-connected nets as hypothetical zinc cyanide polymorphs, as well as their thermal and mechanical properties, through quantum chemical calculations and molecular dynamics simulations. We confirm the metastability of the two porous phases recently discovered experimentally [Lapidus, S. H.; et al. J. Am. Chem. Soc. 2013, 135, 7621-7628], suggest the existence of seven novel porous phases of $\mathrm{Zn}(\mathrm{CN})_{2}$, and show that isotropic negative thermal expansion is a common occurrence among all members of this family of materials, with thermal expansion coefficients close to that of the dense dia-c phase. In constrast, we find a wide variety in the mechanical behavior of these porous structures with framework-dependent anisotropic compressibilities. All porous structures, however, show pressure-induced softening leading to a structural transition at modest pressure.

\section{INTRODUCTION}

Molecular framework materials, including the ubiquitous metal-organic frameworks (MOFs), demonstrate a wide variety of behavior in response to changes in temperature as well as the application of external pressure. ${ }^{1}$ Among the most unusual and high sought-after responses are anomalous thermal and mechanical properties such as negative thermal expansion (NTE), ${ }^{2,3}$ negative linear compressibility (NLC), ${ }^{4,5}$ pressureinduced softening, ${ }^{6}$ and auxeticity (presence of a negative Poisson's ratio in one or more directions). ${ }^{7,8}$ In addition to being rather counterintuitive, these properties have applications in creating zero-thermal expansion composites, artificial muscles, actuators, and blast mitigating materials. In addition, they are of particular interest in nanoporous materials, where their coupling with guest adsorption can be leveraged for sensing applications and in the making of multifunctional materials.

In this context, the prototypical molecular framework zinc cyanide, $\mathrm{Zn}(\mathrm{CN})_{2}$, is a very interesting system. In ambient conditions, it forms a dense interpenetrated structure composed of two diamondoid ( $\mathrm{dia}$ ) frameworks, where the tetrahedral $\mathrm{Zn}^{2+}$ cations are interconnected by linear $\mathrm{Zn}-\mathrm{CN}-$ Zn linkages with disordered cyanide anions. ${ }^{9}$ This dia-c phase of $\mathrm{Zn}(\mathrm{CN})_{2}$ exhibits very strong isotropic NTE, with a volumetric coefficient of thermal expansion of $\alpha_{\mathrm{V}}=-51$ $\mathrm{MK}^{-1}$ as well as extreme pressure-induced softening. ${ }^{10}$ Moreover, it was recently shown that under compression with certain molecular fluids (water, methanol, ethanol) or fluid mixtures, dia-c- $\mathrm{Zn}(\mathrm{CN})_{2}$ can undergo reconstructive transitions to porous noninterpenetrated polymorphs with topologies dia and lon, which are then retained upon release to ambient pressure. $^{11}$

This entirely novel approach to form new porous phases of framework materials raises however several unanswered questions. In particular, do the porous $\mathrm{Zn}(\mathrm{CN})_{2}$ frameworks share the anomalous thermal and mechanical properties of their parent compound, the interpenetrated framework dia-c- $\mathrm{Zn}(\mathrm{CN})_{2}$ ? What is the extent of the influence of the framework topology on the thermal expansion coefficient, the linear compressibility, and their anisotropy? And finally, are there other experimentally feasible porous frameworks in this family, that is, other low-density polymorphs of $\mathrm{Zn}(\mathrm{CN})_{2}$ energetically close to the experimentally observed dia and lon structures?

Here, we select and investigate through quantum chemistry calculations and molecular dynamics simulations a set of frameworks suitable as zinc cyanide polymorphs. First we address the relative stability and structural properties of the corresponding structures at $T=0 \mathrm{~K}$. We then turn to their thermal stability and thermal properties in the 50-500 K range as well as their behavior under isostatic compression and mechanical properties in general.

Received: April 14, 2015

Revised: May 29, 2015

Published: May 29, 2015 
Table 1. List of Nets Studied in This Work, Their Nominal Density, Space Group, Number of Vertices, and Number of Edges. For Each Net, We Also List the Space Group, Density, and Porosity of the Energy-Minimized Zn $(\mathrm{CN})_{2}$ Framework Constructed from It

\begin{tabular}{lccccccccccc}
\multicolumn{1}{c}{ net } & $c f c$ & $c h e$ & dia & dia-c & $g s i$ & lcs & lon & mok & una & unc & unj \\
nominal density & 0.6494 & 0.6842 & 0.6495 & 1.2987 & 0.7384 & 0.6887 & 0.6494 & 1.1116 & 0.6396 & 0.6847 & 0.5989 \\
net space group & $P 6_{3} / m m c$ & $I a \overline{3} d$ & $F d \overline{3} m$ & $P n \overline{3} m$ & $I a \overline{3}$ & $I a \overline{3} d$ & $P 6_{3} / m m c$ & $C c c m$ & $P 6_{1} 22$ & $P 4_{1} 22$ & $P 6_{1} 22$ \\
number of vertices & 2 & 3 & 1 & 1 & 1 & 1 & 1 & 1 & 1 & 1 & 1 \\
number of edges & 3 & 5 & 1 & 1 & 2 & 1 & 2 & 3 & 4 & 2 & 2 \\
framework space group & $\mathrm{P} 6_{3} m c$ & $I 2_{1} 3$ & $R 3 m$ & $P \overline{4} 3 m$ & $I 2_{1} 3$ & $I \overline{4} 3 d$ & $\mathrm{~Pb}_{3} m c$ & $C c$ & $P 6_{1}$ & $P 4_{1}$ & $P 6_{1}$ \\
framework density $\left(\mathrm{g} \mathrm{cm}^{-3}\right)$ & 0.905 & 0.959 & 0.905 & 1.829 & 1.076 & 0.996 & 0.904 & 1.592 & 0.971 & 1.004 & 0.845 \\
porosity & 0.352 & 0.312 & 0.352 & 0.0 & 0.235 & 0.289 & 0.349 & 0.053 & 0.312 & 0.283 & 0.397 \\
\hline
\end{tabular}

\section{SYSTEMS AND METHODS}

II.I. Selection of Frameworks. The use of computational chemistry to study families of polymorphs has long been proven a successful tool for the investigation of structure-property relationships, energetic stability, and experimental feasibility. Seminal work in this area dates back to the early computational studies of all-silica zeolite frameworks with classical force fields ${ }^{12,13}$ and has shown continuing development even in recent years, with focus shifting on a larger number of physical properties ${ }^{14}$ or quantum chemical calculations. ${ }^{15}$ Other families of nanoporous materials have also been studied including the very topical metal-organic frameworks and in particular the family of zeolitic imidazolate frameworks (or ZIFs) ${ }^{16,17}$ This is part of a broader effort at computationally aided discovery of novel materials, through high-throughput screening of candidate materials based on the tools of theoretical chemistry. ${ }^{18,19}$

For this study, we first generated a set of plausible candidates for porous polymorphs of $\mathrm{Zn}(\mathrm{CN})_{2}$ with diverse framework topologies. To that aim, we used the RCSR online database $\mathrm{e}^{20,21}$ of periodic threedimensional nets. We filtered the 2288 nets in the database (at the time of the research) to select those that are structurally close to the known $\mathrm{Zn}(\mathrm{CN})_{2}$ phases, with $\mathrm{Zn}^{2+}$ ions (vertices of the net) tetrahedrally coordinated by cyanide anions (edges). Of the 629 four-coordinated nets in the RCSR database, we selected structures with density in the range of $0.5-1.5 \mathrm{~g} \mathrm{~cm}^{-3}$, near-uniform edge lengths, and $\mathrm{Zn}-\mathrm{Zn}-\mathrm{Zn}$ angles close to $109.5^{\circ}$ (so that the deformations of the $\left[\mathrm{Zn}(\mathrm{CN})_{4}\right]^{2-}$ tetrahedra are not too large).

This search resulted in a set of 11 plausible nets, listed in Table 1 with their nominal density, space group, and number of edges and vertices. This set contains the interpenetrated dense phase dia-c (the only interpenetrated net included) as well as the experimentally known porous phases dia and lon. For each of these nets, we constructed a $\mathrm{Zn}(\mathrm{CN})_{2}$ structure by placing $\mathrm{CN}^{-}$anions along the edges of the net. This ordering of the $\mathrm{CN}^{-}$anions, which are thought to be disordered in the material, was shown by earlier theoretical work to have negligible influence on the structural and dynamic properties of $\mathrm{Zn}(\mathrm{CN})_{2}{ }^{22}$ We thus chose the orientations of the anions so as to obtain for each framework the highest symmetry possible, that is, to be in a subgroup of the net space group with maximal symmetry (Table 1).

II.II. Quantum Chemical Calculations and Molecular Dynamics Simulations. Once the frameworks were chosen, we performed full geometry optimization of the structures using quantum chemistry calculations at the density functional theory (DFT) level, optimizing both atomic positions and unit cell parameters. These DFT calculations were performed using the CRYSTAL14 code, $^{23}$ with localized atomic basis sets, full accounting for the symmetry operators of the crystal, and the B3LYP hybrid exchange-correlation functional. ${ }^{24}$ We used the Grimme "D2" dispersion corrective terms ${ }^{25}$ to effectively take into account long-range (van der Waals) interactions.

Then, to study the influence of temperature and mechanical pressure on the frameworks, we used classical force field-based molecular dynamics simulations in the isostress $(N, \sigma, T)$ ensemble with the DL_POLY software. ${ }^{26}$ The force field used is that derived by Fang et al. for $\mathrm{Zn}(\mathrm{CN})_{2} \cdot{ }^{22}$ It includes (i) a Morse potential governing the distances between a $\mathrm{Zn}$ atom and its $\mathrm{C} / \mathrm{N}$ coordinating neighbors, (ii) several terms creating an energy cost for $\mathrm{C} / \mathrm{N}-\mathrm{Zn}-\mathrm{C} / \mathrm{N}$ and $\mathrm{Zn}-\mathrm{C} / \mathrm{N}-\mathrm{C} / \mathrm{N}$ angles deviating from their values, respectively, $109.5^{\circ}$ and $180^{\circ}$, expected in an ideal tetrahedral geometry, (iii) Coulomb interactions between effective atom-centered point charges that take into account multipolar effects, and (iv) van der Waals interactions described by a Buckingham-type potential. Importantly, given the strength of the $\mathrm{CN}$ bonding, cyanide ions are considered as rigid rods.

For each of the 11 frameworks, a supercell of the crystallographic unit cell obtained by quantum chemistry calculations was selected so that simulation box sizes were close to $40 \AA$ in each direction (in all cases between 30 and $50 \AA$, with aspect ratios as close to 1 as possible). The simulations were performed using the DL_POLY software ${ }^{26}$ in the $(N, \sigma, T)$ ensemble, using a Nosé-Hoover algorithm ${ }^{27}$ allowing anisotropic deformations of the unit cell. Thermostat and barostat relaxation times were set to 1 ps. Equations of motion were integrated using a leapfrog algorithm, with time step of $1 \mathrm{fs}^{28}$ For long-range interactions, the Ewald summation method was used with a precision of $10^{-6}$. Unless specified, the time of each simulation is $200 \mathrm{ps}$, including $20 \mathrm{ps}$ of equilibration. For calculation of elastic stiffness tensors, total simulations times were upped to $5 \mathrm{~ns}$.

\section{ENERGY-MINIMIZED STRUCTURES}

III.I. Quantum Chemical Calculations. We performed energy minimization at the DFT level of the $11 \mathrm{Zn}(\mathrm{CN})_{2}$ frameworks constructed from the RCSR database. Each framework relaxed to a local energy minimum. In all cases, the topology was unchanged during the minimization process: this confirms the metastability of these structures at $0 \mathrm{~K}$. The relative energies of the frameworks are plotted in Figure 1, panel a as a function of density. The dense, interpenetrated dia-c framework, which is the experimental structure of $\mathrm{Zn}(\mathrm{CN})_{2}$ at ambient conditions, is logically found to be the most stable polymorph of $\mathrm{Zn}(\mathrm{CN})_{2}$ studied. The two polymorphs obtained experimentally by fluid intrusion, dia and lon, have a similar energy of $+15 \mathrm{~kJ} / \mathrm{mol}$ with respect to dia-c, in keeping with their near identical structure. They differ mostly in the following aspect: in the dia, all hexagons formed of six neighboring vertices are in chairlike conformation, while in the lon, two-thirds of them are in boat-like conformation; in the corresponding $\mathrm{Zn}(\mathrm{CN})_{2}$ frameworks, this implies differences concerning the relative orientations of neighboring coordination tetrahedra. ${ }^{11}$

In Figure 1, panel a, one can notice a correlation between density and relative energy. This generic feature is widely observed in families of inorganic polymorphs such as zeolites. ${ }^{29}$ Several structures are found within a small energy range of the dia-c framework: these include the experimental structures dia and lon. The $c f c$ framework is very close in energy to the latter two. One structure, the relatively dense $m o k$ ( $\rho=1.592$ $\left.\mathrm{g} \mathrm{cm}^{-3}\right)$, is lower in energy than dia and lon $(\Delta E=12.5 \mathrm{~kJ} /$ $\mathrm{mol})$ due to its relatively high density, close to that of the dia-c structure. Four more structures lie within $20 \mathrm{~kJ} / \mathrm{mol}$ of dia-c 


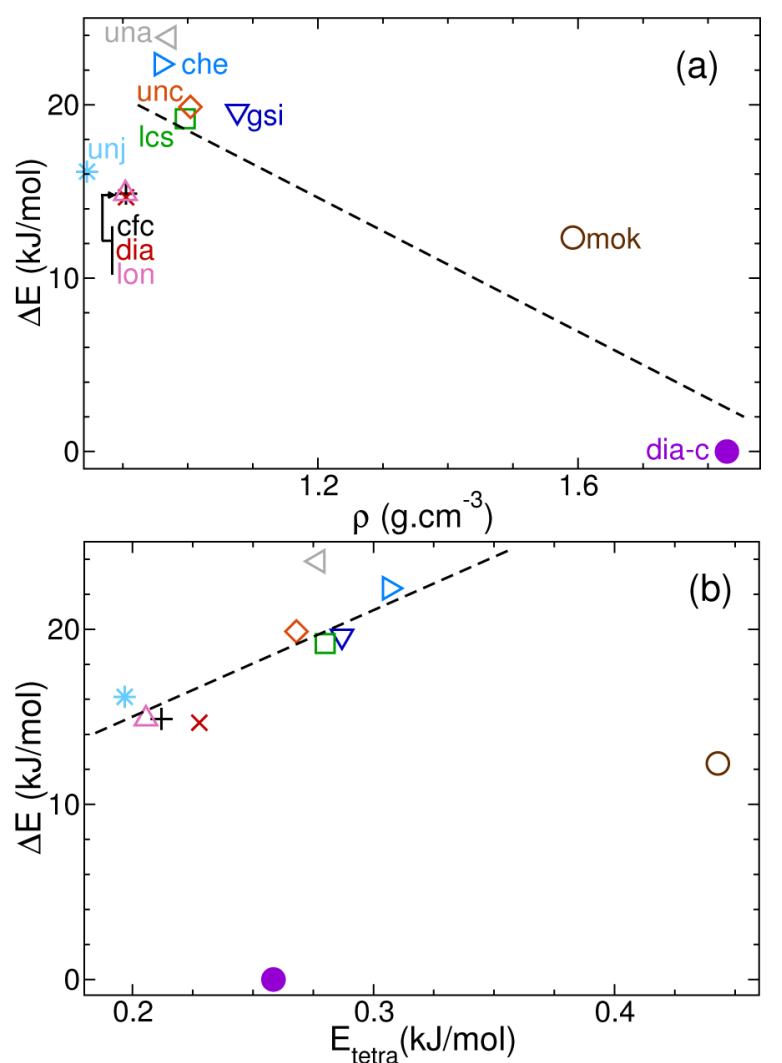

Figure 1. Relative energy of zinc cyanide polymorphs (per $\mathrm{Zn}(\mathrm{CN})_{2}$ unit), using the dense dia-c framework as a reference, as obtained through DFT calculations. (a) Relative energy versus density. (b) Relative energy versus tetrahedral distortion energy (see text for details). Dashed lines are a guide to the eyes, indicating parallel linear trends.

and within $5 \mathrm{~kJ} / \mathrm{mol}$ of the dia and lon: gsi, lcs, unc, and unj. Given the very moderate spread in energy, these structures can thus be considered experimentally feasible. Polymorphs che and una are slightly higher at 22 and $24 \mathrm{~kJ} / \mathrm{mol}$, respectively.

Among the low-density structures studied here, it is worth noting that the lowest energy ones ( $d i a, l o n$, and $c f c$ ) share the same local connectivity and feature quasi-undistorted $\left[\mathrm{Zn}(\mathrm{CN})_{4}\right]^{2-}$ coordination tetrahedra, while the structures with higher energy show larger dispersion in the angles around the $\mathrm{Zn}^{2+}$ ions. To quantify this, we defined a simple energy term $E_{\text {tetra }}$ that quantifies the Coulomb interactions between C/ $\mathrm{N}$ atoms around each $\mathrm{Zn}$ :

$$
E_{\text {tetra }}=\frac{1}{24 \pi \epsilon_{0}}\left\langle\sum_{i<j} \frac{q_{i} q_{j}}{\left|\vec{r}_{i}-\vec{r}_{j}\right|}-\frac{q_{i} q_{j}}{2 d \sin \left(109.47^{\circ}\right)}\right\rangle
$$

where the average is over all $\mathrm{Zn}$ atoms; for each $\mathrm{Zn}$ atom, in eq $1, i$ and $j$ label the four coordinating $\mathrm{C} / \mathrm{N}$ atoms around the central cation; the atomic charges are taken from the force field, and $d$ is the spatially-averaged coordination distance of cations. $^{22}$

The relative energy of the zinc cyanide polymorphs is plotted against $E_{\text {tetra }}$ in Figure 1, panel b. There is a clear correlation, among structures of similar density (i.e., all but mok and dia-c), between tetrahedral distortion and energy. Moreover, we have checked that other factors, such as Coulombic interactions between neighboring $\mathrm{Zn}$ cations, play a minor role in the total energy dependence on topology. The dominant effect in the relative stability of zinc cyanide polymorphs is thus a competition between the distortion of the zinc(II) tetrahedral environment and the topology imposed by the framework for nets whose vertices do not all have a perfect tetrahedral environment. The two exceptions are the dia-c structure, which is stabilized by interframework interactions, and the mok structure, which features very large distortions from the tetrahedral geometry but is also stabilized because of its higher density.

Finally, we calculated the porosity $\phi$ of these structures based on the geometric method of Connolly, ${ }^{30}$ that is, the fraction of the unit cell volume that is accessible to a spherical probe of radius $1.2 \AA^{41}$ The dia-c framework is nonporous, and the mok framework has $\phi=0.053$. Visual inspection reveals that the porosity in mok is composed of small unconnected pockets (closed pores), and it thus has no accessible porosity. All other structures contain open pores and have $0.23<\phi<0.40$, corresponding to estimated pore volumes of $0.253-0.336$ $\mathrm{g} \mathrm{cm}^{-3}$ and surface areas of $3550-3760 \mathrm{~m}^{2} \mathrm{~g}^{-1}$. Among these porous polymorphs, we found a very close correlation between porosity and density (see Figure S4 in Supporting Information) owing to the very similar coordination of these frameworks.

III.II. Force Field Validation. Given our purpose to study these frameworks at finite temperature and pressure using classical force field-based molecular dynamics (MD), one may wonder about the consistency of this approach with the previously discussed DFT calculations, that is, whether the force field validated for the dia-c framework of $\mathrm{Zn}(\mathrm{CN})_{2}$ (both at ambient conditions and under pressure) is also suitable to describe other metastable polymorphs. To check that, we relaxed each polymorph with classical MD in the limit of zero temperature and compared the resulting MD-optimized structures to the DFT-optimized ones. The agreement between structures is found to be excellent: Figure 2 shows that cell

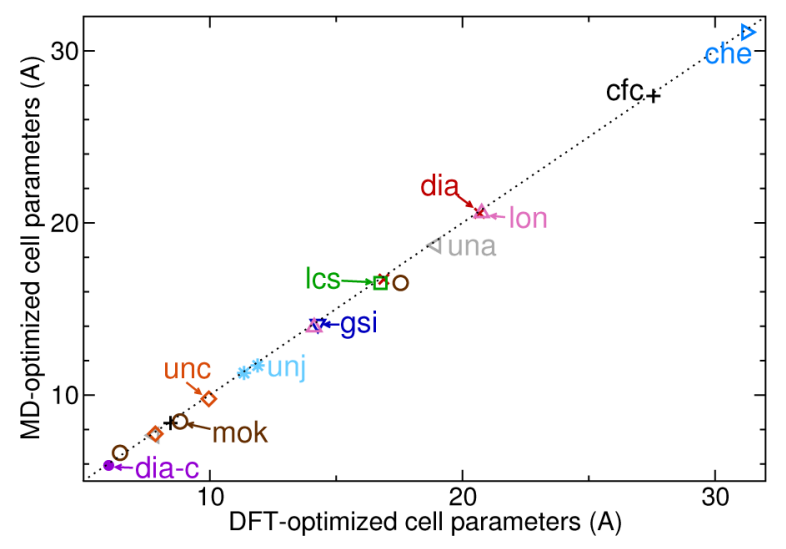

Figure 2. Comparison of DFT- with MD-optimized structures. For a given framework, each cell parameter of the MD-optimized structure and the corresponding parameter of the DFT-optimized structure are given on the $y$ - and $x$-axis, respectively.

parameters show only a tiny variation (a shortening of the order of $1 \%$ ) from the DFT- to the MD-optimized structure. The only exception is the mok structure, where the variation is slightly larger (4\%) but still quite small. Besides, Figure S3 of the Supporting Information shows that the energy differences between these polymorphs are also well reproduced overall. The force field is thus transferable from the dia-c polymorph to the other structures. ${ }^{42}$ 


\section{STABILITY AND THERMAL EXPANSION}

In this section, we describe the thermal properties of $\mathrm{Zn}(\mathrm{CN})_{2}$ polymorphs through molecular dynamics simulations at zero pressure and in a range of temperature from $50-500 \mathrm{~K}$.

Molecular dynamics simulations at increasing values of temperature were used to confirm the metastability of the 11 structures generated. Such confirmation is of particular interest since it was recently shown, for a family of zeolitic imidazolate frameworks with four-connected nets similar to $\mathrm{Zn}(\mathrm{CN})_{2}$, that a large number of the hypothetical polymorphs, which had been proposed based on energy minimizations, ${ }^{16}$ were not thermally stable at room temperature. ${ }^{3}$ While the experimental fluidintruded structures of dia and lon polymorphs of $\mathrm{Zn}(\mathrm{CN})_{2}$ were retained upon release to ambient pressure, and the lon framework appears to retain its structure upon desolvation, ${ }^{11}$ a direct confirmation of these guest-free structures' stability is desirable. It is also unknown whether the same would be true of other potential polymorphs synthesized via fluid intrusion.

All structures were found mechanically stable at ambient temperature and indeed within the whole 50-500 K temperature range. The dia and lon polymorphs obtained experimentally, as well as the other polymorphs, should thus be amenable to fluid evacuation and should retain their porosity upon activation. This indicates the importance of molecular fluid compression as a novel way to obtain new porous structures from dense polymorphs.

We then looked at the thermal behavior of these polymorphs, especially given that the dense dia-c- $\mathrm{Zn}(\mathrm{CN})_{2}$ phase is known for its very strong isotropic NTE. To compare the various polymorphs despite their different crystal symmetries, we plotted the evolutions of unit cell volume $V$ as a function of temperature in Figure 3 and calculated for each

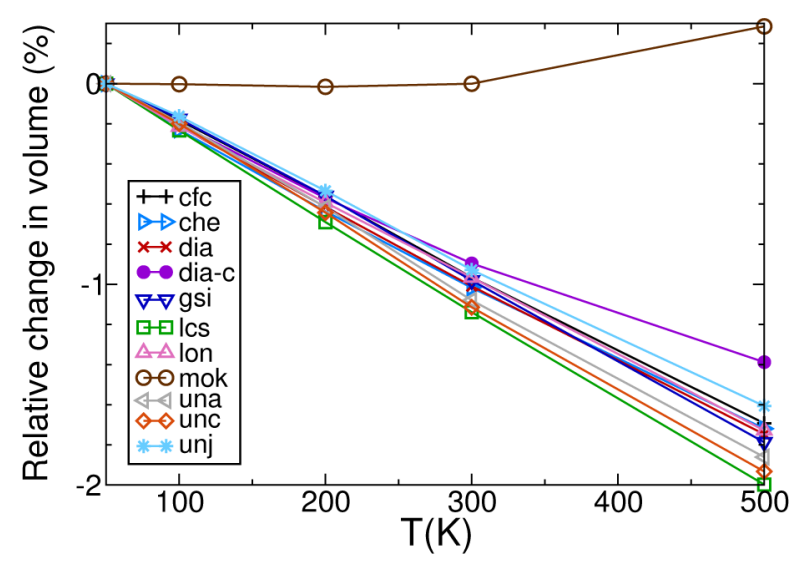

Figure 3. Relative volume change (w.r.t. the value at $50 \mathrm{~K}$ ) versus temperature for the 11 structures of this study.

phase the volume thermal expansion coefficient, $\alpha_{\mathrm{V}}$, in the 50$500 \mathrm{~K}$ range:

$$
\alpha_{\mathrm{V}}=\frac{1}{V}\left(\frac{d V}{d T}\right)
$$

All structures except mok exhibit clear NTE with a roughly linear behavior, very close low-temperature values of $\alpha_{\mathrm{V}}$ (between -35 and $-45 \mathrm{MK}^{-1}$, in reasonable agreement with the experimental value of $-51 \mathrm{MK}^{-1}$ for $\left.d i a-c^{10,31}\right)$. Nonlinearities are mostly noticeable for dense structures: for the dia-c, where these nonlinearities are well described by a quadratic fit, the lessening of NTE under heating is comparable to the one measured in ref $32: \alpha_{\mathrm{V}} \simeq-44 \mathrm{MK}^{-1}$ for $T \rightarrow 0$, while $\alpha_{\mathrm{V}} \simeq-24 \mathrm{MK}^{-1}$ near $400 \mathrm{~K}$. An important feature is that this contraction under heating is essentially isotropic with linear expansion coefficients $\alpha_{a / b / c}$ very close to each other (see Table 1 in Supporting Information).

This near-universality of NTE (and its extent) among the zinc cyanide frameworks, coupled with its isotropic character and its occurrence even in frameworks derived from nonbipartite nets such as $u n j$, indicates that the mechanism at play for NTE does not rely necessarily on rotations of pairs of tetrahedra (involving off-centering of the connecting cyanide) ${ }^{33}$ but can also be compatible with correlated motions of larger units. Consistently, the isotropic character of NTE indicates that it relies on a local, topology-independent mechanism that may well be buckling vibrations of the $\mathrm{Zn}-\mathrm{C}-\mathrm{N}-\mathrm{Zn}$ linkages (as long as tetrahedron distortions are small enough to allow for such vibrations). We provide here an analysis of the buckling vibrations of the $\mathrm{Zn}-\mathrm{C}-\mathrm{N}-\mathrm{Zn}$ linkages, whose importance is clearly demonstrated by the distribution $g(\theta)$ of the angle $\theta$ between a given cyanide axis and the associated $\mathrm{Zn}-\mathrm{Zn}$ axis (see Figure 4). We computed those from the
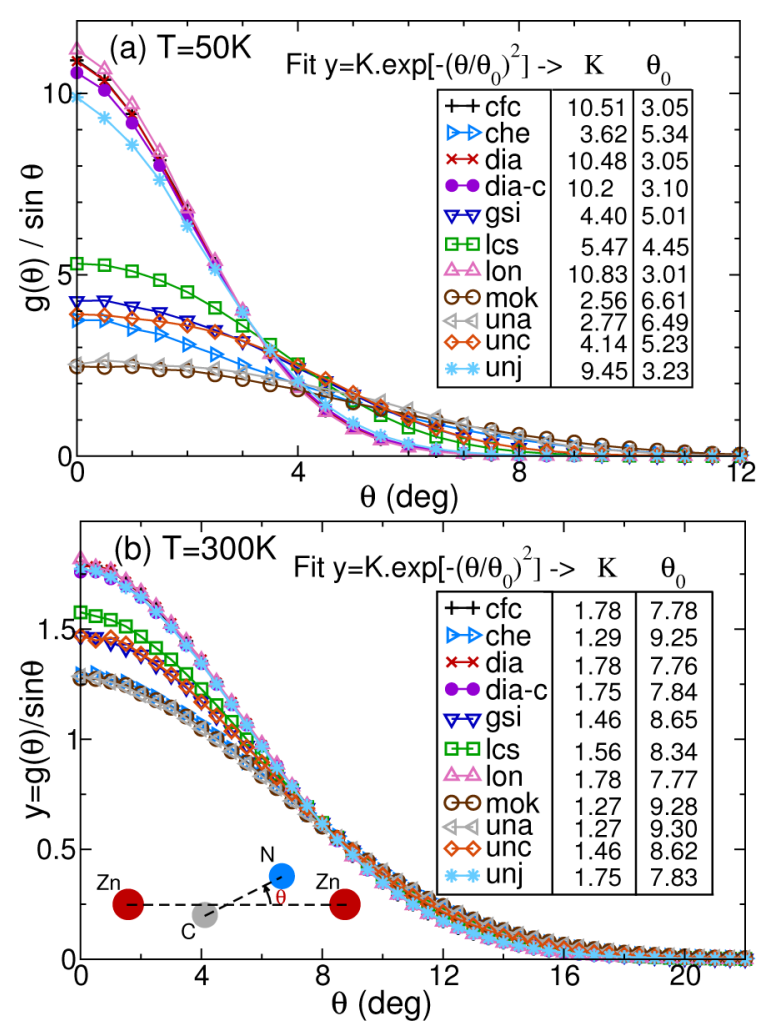

Figure 4. Functions $g(\theta) / \sin \theta$ where $g(\theta)$ is the distribution of angle $\theta$ (see inset of panel $\mathrm{b}$ ), computed for the structures listed, at (a) $T=$ $50 \mathrm{~K}$ and (b) $T=300 \mathrm{~K}$. Gaussian fits were done, with parameters $K$ and $\theta_{0}$ indicated in the table.

atomic trajectory data of $\mathrm{MD}$ simulations at $T=50 \mathrm{~K}$ and $T=$ $300 \mathrm{~K}$. Fits of these distributions indicate clearly a behavior of the form

$$
g(\theta)=K \sin \theta e^{-\left(\theta / \theta_{0}\right)^{2}}
$$

where $K$ is a normalization constant such that $\int_{0}^{(\pi / 2)} g(\theta) d \theta=$ 1 ; the $\sin \theta$ prefactor stands for the solid angle associated with an interval $[\theta ; \theta+d \theta]$. Although the typical angular extension 
$\left(\theta_{0}\right)$ does not depend much on the framework at $T=300 \mathrm{~K}$, at lower temperature, one can distinguish several groups: (i) structures like dia, lon, or unj where $\theta$ in the $T=0 \mathrm{~K}$ structures is negligible ( $\leq 1$ degree), and $\theta_{0}$ is roughly proportional to $\sqrt{ } T$; (ii) structures like mok or una, with large tetrahedron distortion and $\theta$ values exceeding 5 degrees on some bonds of the $T=0$ structure, which significantly enhance $\theta_{0}$ especially at low temperatures; and (iii) intermediate cases. These distributions evidence that, even at $T=50 \mathrm{~K}$, kinetic energy is sufficient for cyanide ions to escape the shallow potential wells resulting from mutually frustrated force fields and to vibrate around the $\mathrm{Zn}-\mathrm{Zn}$ axis. Assuming that these vibrations can combine efficiently to form, at a global scale, rigid unit modes (RUMs) at low energy (which is the case except for the mok), they allow for a large negative $\alpha_{\mathrm{V}}$ value.

The mok structure is here again a particular case: its thermal expansion coefficient is nearly zero. Indeed, distortions of the coordination tetrahedra in the relaxed structure are so large that they hinder tetrahedron rotations and push to higher energy the associated RUMs that would account for NTE otherwise.

\section{MECHANICAL BEHAVIOR}

V.I. Stability under Pressure. After checking that our predicted polymorphs were stable under ambient conditions ( $T$ $=300 \mathrm{~K}, P=0)$, we now address the questions of mechanical stability and behavior under pressure. To this aim, simulations with increasing values of pressure were carried out in a sequential manner, starting from $P=0$ and increasing pressure stepwise by $\Delta P=0.05 \mathrm{GPa}$. Results of these in silico compression experiments are presented in Figure 5 under the

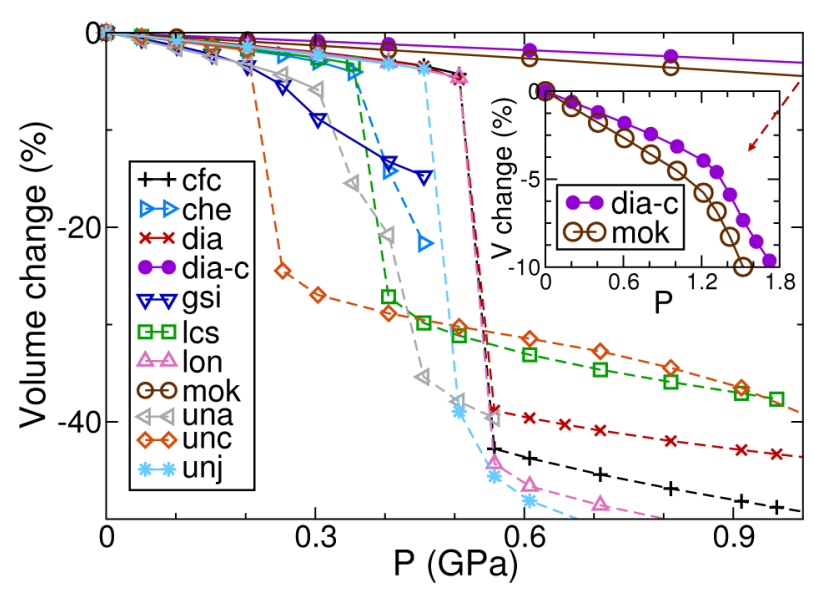

Figure 5. Relative volume change, w.r.t. the zero pressure value, at $T=$ $300 \mathrm{~K}$ and for the 11 structures listed of $\mathrm{Zn}(\mathrm{CN})_{2}$.

form of a volume versus pressure plot. All porous phases have limited stability, undergoing a discontinuous transition at moderate critical pressures ranging from $P_{\mathrm{c}} \simeq 0.2 \mathrm{GPa}($ unc $)$ to $P_{\mathrm{c}} \simeq 0.5 \mathrm{GPa}$ (dia, lon, and $\left.c f c\right)$. The case of the gsi structure stands out somewhat from the others: data up to $0.4 \mathrm{GPa}$ indicate a possible continuous transition near $P \simeq 0.2 \mathrm{GPa}$, but no associated symmetry breaking was evidenced; in any case, the structure was found to be unstable at $P \geq 0.45 \mathrm{GPa}$. Comparison of $P_{c}$ values between different structures indicates that $P_{c}$ clearly increases with the density and for porous structures decreases somewhat with increasing tetrahedral distortions (see Figure S6 in Supporting Information).
This subgigapascal limit of mechanical stability of porous structures contrasts with the behavior of the denser dia-c and mok frameworks: both are stable to pressures exceeding $1 \mathrm{GPa}$, following a generic density/mechanical stability correlation. ${ }^{3}$ The limit of stability of the dia-c structure is found at $P_{c} \simeq 2.1$ GPa. This behavior was already reported in ref 22 and put in parallel with the discontinuous transition toward a highpressure phase, observed experimentally around the same pressure. ${ }^{34}$ Additionally, for both dense structures, a kink in the $V(P)$ curve is found at $P_{\mathrm{c}}^{\prime} \simeq 1.2 \mathrm{GPa}$, reflecting the experimentally observed transition to a high-pressure phase (labeled "phase II" in ref 34). The mok structure shows the same behavior as dia-c under compression. From the analysis of the dia-c and mok trajectories, we can show that this highpressure transformation involves a shortening of the distances between a zinc and its nearest noncoordinating cyanides (see Figure S5 of the Supporting Information and accompanying text), hinting to a move toward higher coordination around the zinc, as evidenced experimentally by Collings et al. ${ }^{34}$

The behavior of porous structures under compression can also be analyzed by considering the distributions of the cyanide's buckling angle, $\theta$ (see Figure 4 ). In the low-pressure phases (for $P<P_{c}$ ), the distributions $g(\theta)$, shown in Figures S8-S10 of the Supporting Information, still follow the law given in eq 3 . The typical angular extension $\theta_{0}$ increases with pressure, as increasing $\theta$ allows to decrease cell parameters without decreasing the distances between $\mathrm{Zn}$ and its coordinating atoms. In the high-pressure phases of porous structures, this type of distributions is also observed; however, the $\theta_{0}(P)$ plots are characterized by a jump at $P_{\mathrm{c}}$. In fact, the transition is better characterized by (i) the distribution $g(d)$ of the distance $d$ between a given cyanide ion and the associated $\mathrm{Zn}-\mathrm{Zn}$ axis (see inset of Figure 6c), or (ii) the radial distributions $g\left(r_{\mathrm{Zn}-\mathrm{X}}\right)$ (either $\mathrm{X}=\mathrm{Zn}$ for $\mathrm{Zn}-\mathrm{Zn}$ distances or $\mathrm{X}$ $=\mathrm{C} / \mathrm{N}$ for distances between $\mathrm{Zn}$ and either $\mathrm{C}$ or $\mathrm{N}$ ).

In Figure 6, for the porous structures dia and lcs, one can clearly distinguish the unimodal distributions $g(d)$ in the lowpressure phase from those in the high-pressure phase, which are bimodal, indicating the coexistence in this phase of two types of $\mathrm{Zn}-\mathrm{CN}-\mathrm{Zn}$ linkages, one of them being much more distorted than the other. The case of the dense dia-c is different and consistent with a transition of a continuous nature. At $P_{\mathrm{c}}^{\prime} \simeq 1.2$ $\mathrm{GPa}$, the distribution remains monomodal, but one notices a clear change of behavior: the distribution extension (or $\langle d\rangle)$ is almost unaffected by $P$ in the low-pressure regime, while for $P>$ $P_{\mathcal{c}}^{\prime}$, it increases much faster. Although $\langle d\rangle$ cannot strictly be considered as an order of parameter for this transition (it is nonzero in the low-pressure phase), its behavior supports the picture of a continuous transition induced by a specific phonon mode softening, and involving cyanide ions off-centering from their respective $\mathrm{Zn}-\mathrm{Zn}$ axes. This conclusion can also be reached by the analysis of the distributions of radial distances $g\left(r_{\mathrm{Zn}-\mathrm{C} / \mathrm{N}}\right)$ (see Figure S5 of the Supporting Information and accompanying text).

V.II. Response in the Low-Pressure Regime. We now turn our attention to the mechanical properties of the structures predicted in this work. For each framework, we performed a fit of the volume-pressure curve by a third-order Birch-Murnaghan equation of state $^{35}$ in the low-pressure region: 

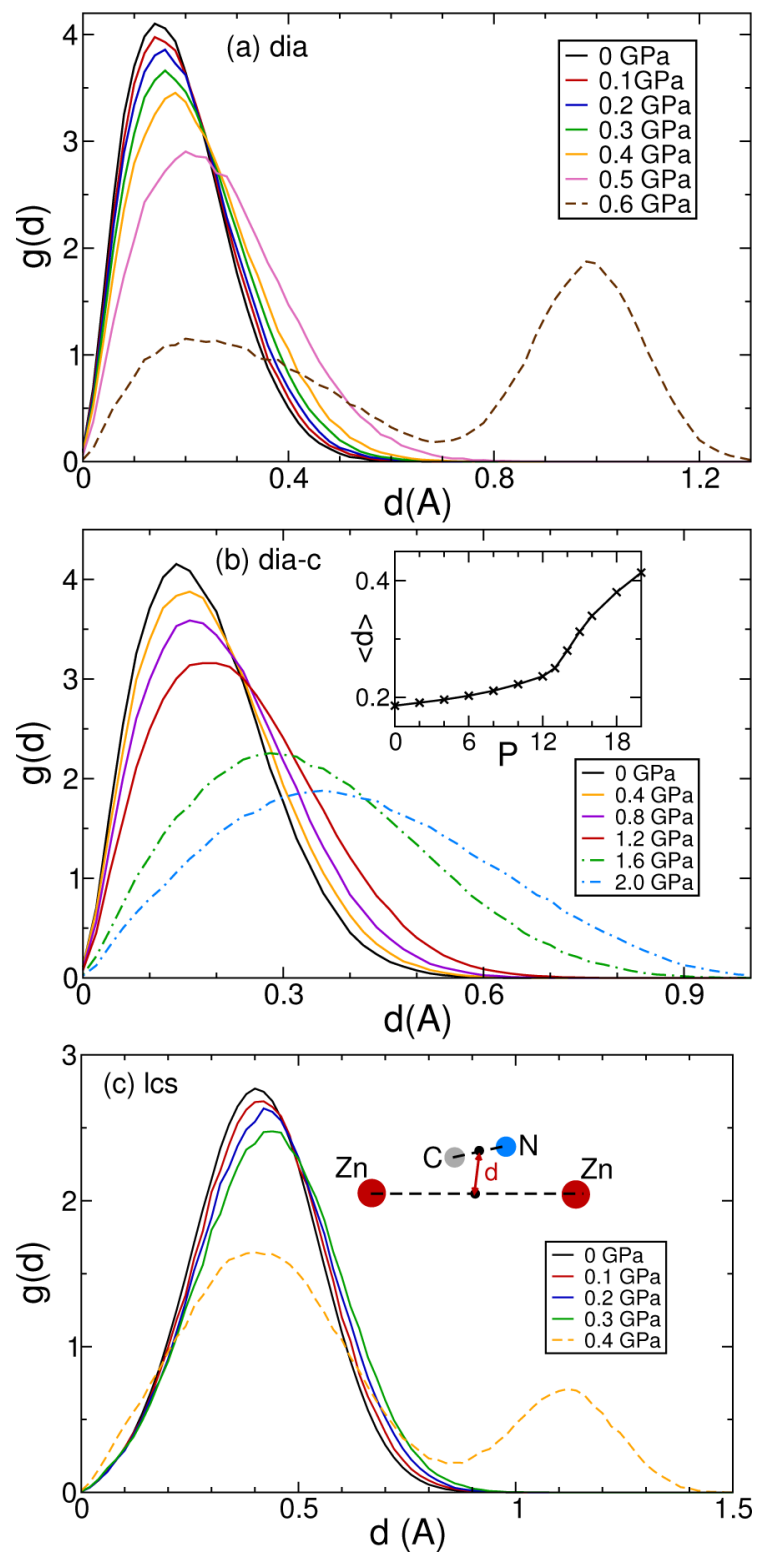

Figure 6. Distributions of the distance $d$ (in ångströms) between (the center of mass of) a $\mathrm{CN}$ ion and the middle of the associated $\mathrm{Zn}-\mathrm{Zn}$ linkage for the dia (a), dia-c (b), and lcs (c) structures. $g(d)$ was computed from atomic trajectory data of $\mathrm{MD}$ simulations at $T=300$ $\mathrm{K}$, and pressures $P$ indicated in the captions. For dia-c, the inset shows the evolution of the average distance $\langle d\rangle$ with pressure.

$$
\begin{aligned}
P(V)= & \frac{3 B_{0}}{2}\left[\left(\frac{V_{0}}{V}\right)^{7 / 3}-\left(\frac{V_{0}}{V}\right)^{5 / 3}\right] \\
& \times\left[1+\frac{3}{4}\left(B_{0}^{\prime}-4\right)\left(\left(\frac{V_{0}}{V}\right)^{2 / 3}-1\right)\right]
\end{aligned}
$$

to obtain zero-pressure values of bulk modulus, $B_{0}=B(P=0)$, and of its derivative with respect to pressure, $B_{0}^{\prime}=(d B / d P)(P=$ $0)$. Bulk moduli show much dispersion among the 11 structures considered (Figure 7$)$. The largest $\left(B_{0}=34.6 \mathrm{GPa}\right)$ is found for the densest dia-c structure. In fact, we observe a good correlation between $B_{0}$ and other structural factors, in particular, the density $\rho$ and the relative energy $\Delta E$. Moreover, among the porous polymorphs, we find a close correlation
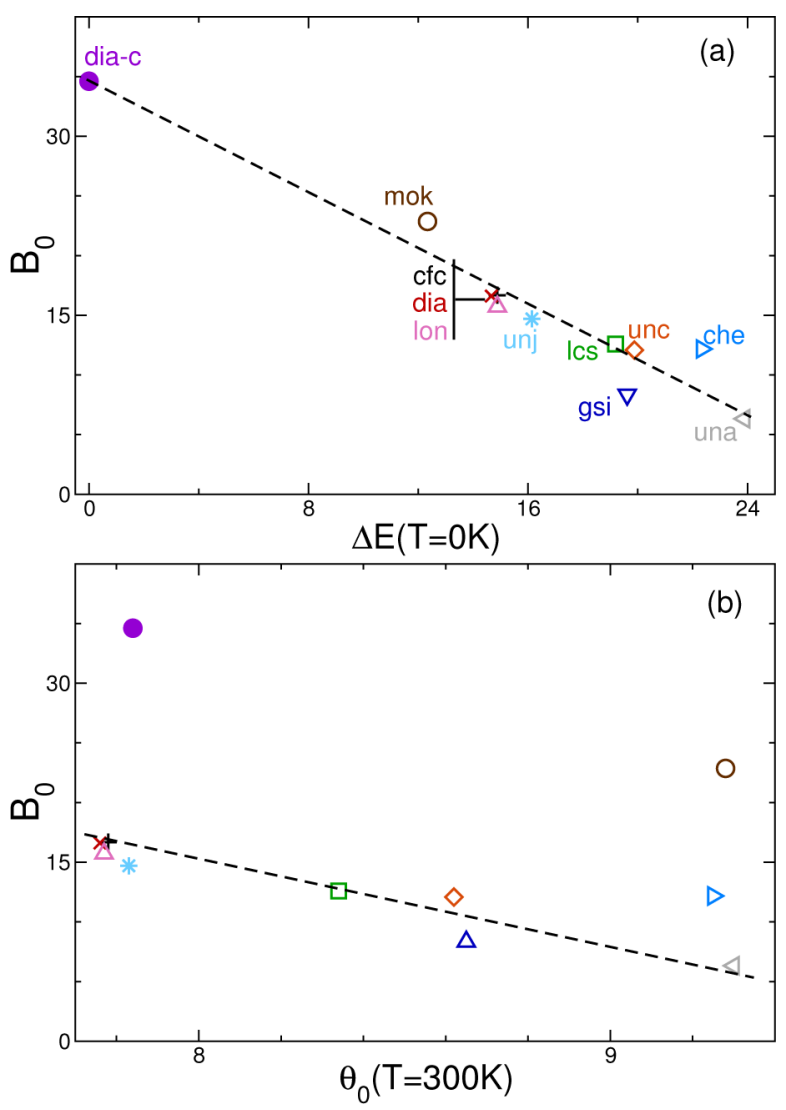

Figure 7. Bulk moduli $B_{0}$ (in GPa) estimated from MD simulations at $T=300 \mathrm{~K}$ and subsequent fits to eq 4 for the 11 structures indicated; they are plotted against (a) the DFT-obtained energy $\Delta E(T=0 \mathrm{~K})$ (in $\mathrm{kJ} / \mathrm{mol}$ ) and (b) the angle $\theta_{0}$ (in degrees).

between the bulk modulus and the amplitude of buckling modes $\theta_{0}($ at $300 \mathrm{~K})$. Finally, we also observe that the stiffest structures (high $B_{0}$ ) feature higher resistance to pressure (see Figure S6c in Supporting Information).

All polymorphs studied here have a negative value of $B_{0}^{\prime}$ (see Figure S7 in Supporting Information), reflecting an anomalous decrease in bulk modulus with increasing pressure or pressureinduced softening. ${ }^{36}$ This behavior, first demonstrated in the dia-c phase in ref $10\left(B_{0}^{\prime}=-6.0(7)\right)$ and later measured in various pressure-transmitting media $\left(B_{0}^{\prime}=-8.6(13)\right.$ in ref 34; $B_{0}^{\prime}=-8.48(15)$ in ref 11$)$, is apparently a universal feature of $\mathrm{Zn}(\mathrm{CN})_{2}$ polymorphs. Our force field-based simulations show only qualitative agreement with those experimental measurements, with a value of $B_{0}^{\prime}=-4.0$ for dia-c. Nevertheless, this allows us to compare the extent of softening between the structures studied. We find that the value of $B_{0}^{\prime}$ obtained for the porous polymorphs is much larger than that of dia-c, with values around -10 for the $d i a$, lon, $c f c$, and $u n c$ phases and -16 for gsi. This is particularly large compared to known minerals exhibiting pressure-induced softening such as malayaite $\left(B_{0}^{\prime}=\right.$ $-3)$. From comparing values of $B_{0}^{\prime}$ in different structures, it seems that $B_{0}^{\prime}$ is less negative for dense or highly distorted structures (dia-c, mok, una), but no simple relationship could be established between $B_{0}^{\prime}$ and any of the frameworks' physical properties (density, relative energy, etc.). While the existence of universal pressure-induced softening in the $\mathrm{Zn}(\mathrm{CN})_{2}$ family is inherent to the chemistry of the $\mathrm{Zn}-\mathrm{C}-\mathrm{N}-\mathrm{Zn}$ linkages, and linked to its universally shared anomalous thermal expansion, its extent is dependent on the structure's symmetries via the 
Table 2. Minimal and Maximal Values, For Each Structure at $T=300 \mathrm{~K}$, of Linear Compressibility $\beta$ (in $\mathrm{TPa}^{-1}$ ), Young's Modulus E (in GPa), Shear Modulus G (in GPa), and Poisson's Ratio

\begin{tabular}{|c|c|c|c|c|c|c|c|c|}
\hline phase & $\beta_{\min }$ & $\beta_{\max }$ & $E_{\min }$ & $E_{\max }$ & $G_{\min }$ & $G_{\max }$ & $\nu_{\min }$ & $\nu_{\text {max }}$ \\
\hline$c f c$ & 19.6 & 22.4 & 16.0 & 25.2 & 1.6 & 13.2 & -0.32 & 1.02 \\
\hline che & 26.3 & 28.6 & 6.2 & 34.7 & 2.17 & 17.4 & -0.53 & 1.09 \\
\hline dia & 19.1 & 22.9 & 11.1 & 30.9 & 3.5 & 17.1 & -0.22 & 0.86 \\
\hline $\operatorname{dia}-c$ & 9.3 & 10.0 & 9.1 & 83.3 & 3.6 & 50.2 & -0.65 & 1.31 \\
\hline$g s i$ & 37.7 & 40.4 & 8.5 & 27.4 & 3.3 & 15.5 & -0.40 & 0.73 \\
\hline lcs & 24.6 & 26.9 & 8.6 & 21.9 & 3.2 & 10.6 & -0.18 & 0.75 \\
\hline lon & 18.5 & 28.0 & 15.0 & 24.8 & 1.2 & 13.1 & -0.51 & 1.24 \\
\hline mok & 11.3 & 20.8 & 18.8 & 55.6 & 4.6 & 29.3 & -0.32 & 0.95 \\
\hline una & -5.7 & 10.4 & 8.4 & 23.2 & 0.60 & 18.7 & -0.96 & 1.63 \\
\hline unc & 6.7 & 40.6 & 9.3 & 23.1 & 2.8 & 20.3 & -0.41 & 1.05 \\
\hline$u n j$ & 19.1 & 34.7 & 7.1 & 26.1 & 0.68 & 13.8 & -0.81 & 1.53 \\
\hline
\end{tabular}

nature and repartition in $\mathbf{k}$-space of rigid unit modes accounting for it. ${ }^{22,37}$

V.III. Mechanical Properties. There has been a lot of interest recently in the search for framework materials with anomalous mechanical properties including negative linear compressibility, ${ }^{4,5}$ negative Poisson's ratio, ${ }^{7,8}$ and highly anisotropic mechanical behavior. ${ }^{5}$ We have thus further quantified the mechanical response of the proposed $\mathrm{Zn}(\mathrm{CN})_{2}$ polymorphs by carrying out long MD runs (total time: $5 \mathrm{~ns}$ ) at $T=300 \mathrm{~K}$ and $P=0$ for each of the 11 structures. From the fluctuations of cell parameters, their stiffness tensors $C_{i j}(1 \leq i, j$ $\leq 6$ in Voigt notation) can be extracted:

$$
C_{i j}^{-1}=\left(\frac{V}{k_{\mathrm{B}} T}\right)\left(\left\langle\epsilon_{i} \epsilon_{j}\right\rangle-\left\langle\epsilon_{i}\right\rangle\left\langle\epsilon_{j}\right\rangle\right)
$$

(with $\epsilon$ the strain tensor). These in turn give access to various direction-dependent quantities such as the linear compressibility $\beta(\mathbf{u})$, the Young's modulus $E(\mathbf{u})$, or the shear modulus $G(\mathbf{u}, \mathbf{v})$, which quantify, respectively, the deformation along $\mathbf{u}$ subsequent to a uniform compression, the deformation along $\mathbf{u}$ following a compression along this axis, and the resistance of the plane $\mathcal{P}$ normal to $\mathbf{v}$ to shearing along $\mathbf{u} \in \mathcal{P}^{5}$ (Table 2). We used the ELATE online tool to perform this analysis. ${ }^{38}$

Concerning the linear compressibility, only one structure exhibits negative linear compressibility: the una, which expands slightly along one direction (the $c$ axis) upon isostatic compression: $\beta(\mathrm{c}) \simeq-5 \mathrm{TPa}^{-1}$. The $c$ axis in this structure is that of porous channels; unlike the wine-rack structure of some MOFs, ${ }^{39}$ where $1 \mathrm{D}$ porous channels along a given axis allow for a large NLC in a direction transverse to channels; here the six-fold symmetry of the channel lattice disables this effect. Yet, this small NLC along $c$ is reminiscent of the very small compressibility along the channel direction in the wine-rack case. Other structures with well-defined linear channels have also large anisotropy in linear compressibility, for example, the unc has $\beta(\mathrm{c})=6.7 \mathrm{TPa}^{-1} \simeq \beta_{\text {in-plane }} / 6$. In contrast, structures for which the framework is of cubic symmetry, or at least contains distinct types of channels with noncollinear axes, have much smaller anisotropy in linear compressibility, $\beta_{\max } / \beta_{\min } \leq$ 1.2 .

All structures show substantial anisotropy in Young's moduli. The anisotropy ratio $E_{\max } / E_{\min }$ varies from values $\simeq 1.6$ ( $c f c$ and lon structures) to 9.1 for the dia-c. The latter value can appear large for a highly symmetric and dense structure. In fact, there, the Young's modulus is maximized along the [111] direction (83.3 GPa) and those equivalent by symmetry; compression along such an axis, due to the interpenetration of frameworks, would necessarily involve compression of $\mathrm{Zn}-\mathrm{C}-\mathrm{N}-\mathrm{Zn}$ linkages along this axis. Thus, the material is much stiffer along these directions than along crystallographic axes like [100], along which compression can occur by, for example, deforming coordination tetrahedra and without compressing individual bonds. For porous structures, where void space allows more easily for compression by angular distortions, the maximal Young's modulus is much smaller (reaching 34.7 GPa somehow for the che structure). The minimal Young's modulus can be as small as 6.2 and $7.1 \mathrm{GPa}$ for the che and unj, respectively. In the latter case, it corresponds to a compression along $1 \mathrm{D}$ channels, which is probably easier since the walls of these channels are made of edge-sharing pentagons (each edge consisting of a $\mathrm{Zn}-\mathrm{C}-\mathrm{N}-\mathrm{Zn}$ linkage, and none of these edges is along the channel axis) and thus relatively easy to deform. The che case, though with a more complex structure, also allows easy compression along certain axes, forming substantial angles $\geq 30$ degrees with all $\mathrm{Zn}-\mathrm{C}-\mathrm{N}-\mathrm{Zn}$ linkages of the framework.

The response to uniaxial stress is also characterized by the Poisson's ratio $\nu(\mathbf{u}, \mathbf{v})$ (ratio of tranvserse strain along $\mathbf{v}$ to strain along the compression axis $\mathbf{u}$ ). Remarkably, we find this ratio to be negative in some directions for all the structures studied. The most negative values are found (i) for structures with well-defined $1 \mathrm{D}$ channels like una or unj; (ii) the dia-c structure. Auxeticity in these materials seem to result from a mechanism already encountered in zeolite frameworks involving correlated rotations of neighboring tetrahedra. ${ }^{8}$

Finally, to confirm the nature of the pressure-induced phase transition in porous phases of $\mathrm{Zn}(\mathrm{CN})_{2}$, we performed calculations of elastic constants of the dia structure at increasing values of pressure up to $P_{c}$. The bulk moduli, $B(P)$, calculated at each pressure from the elastic tensors, is shown in Figure 8. It can be seen to decrease with pressure, following a power law $\approx$ $\left(P_{\mathrm{c}}-P\right)^{\alpha}$, with exponent $\alpha \simeq 0.36$. Moreover, when approaching the transition, the lowest eigenvalues of the stiffness matrix (see Figure 8) decrease significantly; an analysis of the associated eigenvectors gives indication for a shearing mode, stimulated by compression along a transverse axis (i.e., noncollinear to cell vectors $\mathbf{T}_{a / b / c}$ ). This demonstrates that the instability under pressure is due to pressure-induced shear mode softening, similar to that observed for several porous MOFs. ${ }^{3,40}$

\section{CONCLUSIONS}

Following the recent report that $\mathrm{Zn}(\mathrm{CN})_{2}$ can undergo reconstructive transitions to porous polymorphs upon 


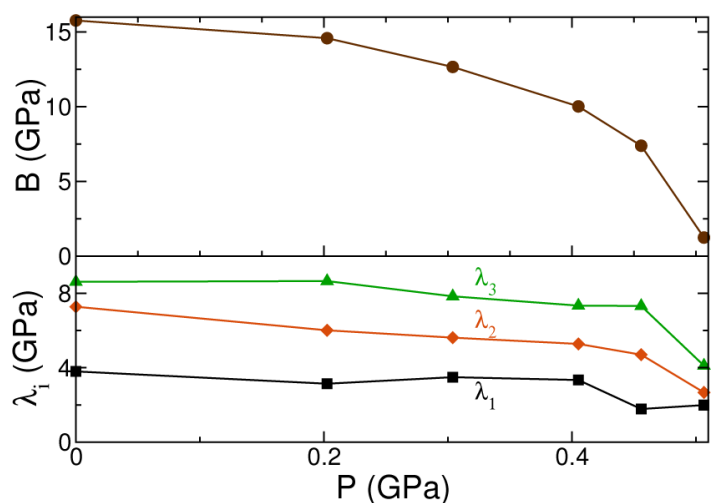

Figure 8. Mechanical properties of the dia framework versus pressure, studied by $10 \mathrm{~ns}$-long MD runs, at $T=300 \mathrm{~K}$. Up, bulk modulus $B(P)$; down, three lowest eigenvalues $\lambda_{i}$ of the $6 \times 6$ stiffness tensor.

compression in molecular fluids, we have investigated the feasibility of ten different four-connected nets as hypothetical zinc cyanide polymorphs. Through a combination of quantum chemical calculations and molecular dynamics simulations, we have confirmed the metastability of the two phases recently evidenced experimentally and suggest the existence of seven novel porous phases of $\mathrm{Zn}(\mathrm{CN})_{2}$ with relatively small formation enthalpy from the dense polymorph. Furthermore, we have characterized their thermal and mechanical behavior, showing that isotropic negative thermal expansion is a nearuniversal feature of this family of materials, with thermal expansion coefficients somewhat larger than that of the dense phase. In constrast, we find a wide variety in the mechanical behavior of these porous structures, with framework-dependent anisotropic compressibilities. All porous structures, however, show pressure-induced softening leading to a structural transition at modest pressure.

\section{ASSOCIATED CONTENT}

\section{(S Supporting Information}

DFT-optimized structures; input files for DFT and molecular dynamics calculations; representations of the frameworks studied; table of linear and volumetric thermal expansion coefficients; values of minimal and maximal $\mathrm{Zn}-\mathrm{Zn}-\mathrm{Zn}$ angles; plots showing energy and porosity versus density (at $T=0 \mathrm{~K}$ and $P=0 \mathrm{GPa}$ ); distributions of distances for various pressures in two frameworks; plots showing the critical pressure $P_{\mathrm{c}}$ and the coefficient $B_{0}^{\prime}$ at $T=300 \mathrm{~K}$; distributions of angles $\theta$ at various pressures and evolution of cell parameters versus $P$ (at $T=300 \mathrm{~K})$. The Supporting Information is available free of charge on the ACS Publications website at DOI: 10.1021/ acs.chemmater.5b01366.

\section{AUTHOR INFORMATION}

\section{Corresponding Authors}

*E-mail: anne.boutin@ens.fr.

*Email: fx.coudert@chimie-paristech.fr. Twitter: @fxcoudert. Web: http://coudert.name/.

\section{Notes}

The authors declare no competing financial interest.

\section{ACKNOWLEDGMENTS}

We thank Aurélie Ortiz, Alain Fuchs, and Andrew Goodwin for helpful comments and discussions. We acknowledge the access to HPC resources from GENCI (Grant No. x2015087069).

\section{REFERENCES}

(1) Coudert, F.-X. Responsive Metal-Organic Frameworks and Framework Materials: Under Pressure, Taking the Heat, in the Spotlight, with Friends. Chem. Mater. 2015, 27, 1905-1916.

(2) Evans, J. S. O.; Mary, T. A.; Vogt, T.; Subramanian, M. A.; Sleight, A. W. Negative Thermal Expansion in $\mathrm{ZrW}_{2} \mathrm{O}_{8}$ and $\mathrm{HfW}_{2} \mathrm{O}_{8}$. Chem. Mater. 1996, 8, 2809-2823.

(3) Bouëssel du Bourg, L.; Ortiz, A. U.; Boutin, A.; Coudert, F. X. Systematic Investigation of Thermal and Mechanical Stability of Zeolitic Imidazolate Frameworks (ZIF). APL Mater. 2014, 2, 124110.

(4) Cairns, A. B.; Thompson, A. L.; Tucker, M. G.; Haines, J.; Goodwin, A. L. Rational Design of Materials with Extreme Negative Compressibility: Selective Soft-Mode Frustration in $\mathrm{KMn}\left[\mathrm{Ag}(\mathrm{CN})_{2}\right]_{3}$. J. Am. Chem. Soc. 2012, 134, 4454-4456.

(5) Ortiz, A. U.; Boutin, A.; Fuchs, A.; Coudert, F.-X. Metal-Organic Frameworks with Wine-Rack Motif: What Determines Their Flexibility and Elastic Properties? J. Chem. Phys. 2013, 138, 174703174710.

(6) Pantea, C.; et al. Pressure-Induced Elastic Softening of Monocrystalline Zirconium Tungstate at $300 \mathrm{~K}$. Phys. Rev. B 2006, 73,214118

(7) Grima, J. N.; Gatt, R.; Alderson, A.; Evans, K. E. On the Origin of Auxetic Behavior in the Silicate $\alpha$-Cristobalite. J. Mater. Chem. 2005, $15,4003-4005$.

(8) Grima, J. N.; Zammit, V.; Gatt, R.; Alderson, A.; Evans, K. E. Auxetic Behavior from Rotating Semi-Rigid Units. Phys. Status Solidi B 2007, 244, 866-882.

(9) Williams, D. J.; Partin, D. E.; Lincoln, F. J.; J. Kouvetakis, J.; O' Keefe, M. The Disordered Crystal Structures of $\mathrm{Zn}(\mathrm{CN})_{2}$ and $\mathrm{Ga}(\mathrm{CN})_{3}$. J. Solid State Chem. 1997, 134, 164-169.

(10) Chapman, K. W.; Chupas, P. J. Pressure Enhancement of Negative Thermal Expansion Behavior and Induced Framework Softening in Zinc Cyanide. J. Am. Chem. Soc. 2007, 129, 10090-10091.

(11) Lapidus, S. H.; et al. Exploiting High Pressures To Generate Porosity, Polymorphism, and Lattice Expansion in the Nonporous Molecular Framework $\mathrm{Zn}(\mathrm{CN})_{2}$. J. Am. Chem. Soc. 2013, 135, 76217628.

(12) Henson, N. J.; Cheetham, A. K.; Gale, J. D. Theoretical Calculations on Silica Frameworks and Their Correlation with Experiment. Chem. Mater. 1994, 6, 1647-1650.

(13) De Boer, K.; Jansen, A. P. J.; Van Santen, R. A. StructureStability Relationships for All-Silica Structures. Phys. Rev. B: Condens. Matter Mater. Phys. 1995, 52, 12579-12590.

(14) Bushuev, Y. G.; Sastre, G. Feasibility of Pure Silica Zeolites. J. Phys. Chem. C 2010, 114, 19157-19168.

(15) Coudert, F.-X. Systematic Investigation of the Mechanical Properties of Pure Silica Zeolites: Stiffness, Anisotropy, and Negative Linear Compressibility. Phys. Chem. Chem. Phys. 2013, 15, 1601216018.

(16) Lewis, D. W.; Ruiz-Salvador, A. R; Gomez, A.; RodriguezAlbelo, L. M.; Coudert, F.-X.; Slater, B.; Cheetham, A. K.; MellotDraznieks, C. Zeolitic Imidazole Frameworks: Structural and Energetics Trends Compared with Their Zeolite Analogues. CrystEngComm 2009, 11, 2272-2276.

(17) Baburin, I. A.; Leoni, S. Modelling Polymorphs of MetalOrganic Frameworks: A Systematic Study of Diamondoid Zinc Imidazolates. CrystEngComm 2010, 12, 2809-2816.

(18) Watanabe, T.; Sholl, D. S. Accelerating Applications of MetalOrganic Frameworks for Gas Adsorption and Separation by Computational Screening of Materials. Langmuir 2012, 28, 1411414128.

(19) Wilmer, C. E.; Leaf, M.; Lee, C. Y.; Farha, O. K.; Hauser, B. G.; Hupp, J. T.; Snurr, R. Q. Large-Scale Screening of Hypothetical Metal-Organic Frameworks. Nat. Chem. 2012, 4, 83-89.

(20) Reticular Chemistry Structure Resource, 2008. http://rcsr.net/nets (accessed June 5, 2015).

(21) O'Keeffe, M.; Peskov, M. A.; Ramsden, S. J.; Yaghi, O. M. The Reticular Chemistry Structure Resource (RCSR) Database of, and Symbols for, Crystal Nets. Acc. Chem. Res. 2008, 41, 1782-1789. 
(22) Fang, H.; Dove, M. T.; Rimmer, L. H. N.; Misquitta, A. J. Simulation Study of Pressure and Temperature Dependence of the Negative Thermal Expansion in $\mathrm{Zn}(\mathrm{CN})_{2}$. Phys. Rev. B 2013, 88, 104306.

(23) Dovesi, R.; Orlando, R.; Civalleri, B.; Roetti, C.; Saunders, V. R.; Zicovich-Wilson, C. M. CRYSTAL: A Computational Tool for the $a b$ Initio Study of the Electronic Properties of Crystals. Z. Kristallogr. 2005, 220, 571-573.

(24) Becke, A. D. Density-Functional Thermochemistry III. The Role of Exact Exchange. J. Chem. Phys. 1993, 98, 5648.

(25) Grimme, S. Semiempirical GGA-Type Density Functional Constructed with a Long-Range Dispersion Correction. J. Comput. Chem. 2006, 27, 1787-1799.

(26) Todorov, I. T.; Smith, W.; Trachenko, K.; Dove, M. T. DL

POLY 3: New Dimensions in Molecular Dynamics Simulations via Massive Parallelism. J. Mater. Chem. 2006, 16, 1911-1918.

(27) Hoover, W. G. Canonical Dynamics: Equilibrium Phase-Space Distributions. Phys. Rev. A 1985, 31, 1695-1697.

(28) Allen, M. P.; Tildesley, D. J. Computer Simulation of Liquids; Clarendon Press: Oxford, 1989; pp 5, 49, 58, 60, 63, 179, 181.

(29) Zwijnenburg, M. A.; Illas, F.; Bromley, S. T. Apparent Scarcity of Low-Density Polymorphs of Inorganic Solids. Phys. Rev. Lett. 2010, 104, 175503.

(30) Connolly, M. L. Solvent-Accessible Surfaces of Proteins and Nucleic Acids. Science 1983, 221, 709-713.

(31) Goodwin, A.; Kepert, C. J. Negative Thermal Expansion and Low-Frequency Modes in Cyanide-Bridged Framework Materials. Phys. Rev. B 2005, 71, R140301.

(32) Chapman, K. W.; Chupas, P. J.; Kepert, C. J. Direct Observation of a Transverse Vibrational Mechanism for Negative Thermal Expansion in $\mathrm{Zn}(\mathrm{CN})_{2}$ : An Atomic Pair Distribution Function Analysis. J. Am. Chem. Soc. 2005, 127, 15630-15636.

(33) Goodwin, A. Rigid Unit Modes and Intrinsic Flexibility in Linearly Bridged Framework Structures. Phys. Rev. B 2006, 74, 134302-134311.

(34) Collings, I.; et al. Homologous Critical Behavior in the Molecular Frameworks $\mathrm{Zn}(\mathrm{CN})_{2}$ and $\mathrm{Cd}(\text { imidazolate })_{2}$. J. Am. Chem. Soc. 2013, 135, 7610.

(35) Birch, F. Finite Elastic Strain of Cubic Crystals. Phys. Rev. 1947, $71,809$.

(36) Fang, H.; Philipps, A. E.; Dove, M. T.; Tucker, M. G.; Goodwin, A. L. Temperature-Dependent Pressure-Induced Softening in $\mathrm{Zn}$ (CN) $)_{2}$. Phys. Rev. B 2013, 88, 144103.

(37) Fang, H.; Dove, M. T. Pressure-Induced Softening as a Common Feature of Framework Structures with Negative Thermal Expansion. Phys. Rev. B 2013, 87, 214109-214114.

(38) Coudert, F.-X. ELATE: Elastic tensor analysis, 2013. http:// progs.coudert.name/elate (accessed June 5, 2015).

(39) Ortiz, A. U.; Boutin, A.; Fuchs, A.; Coudert, F.-X. Anisotropic Elastic Properties of Flexible Metal-Organic Frameworks: How Soft Are Soft Porous Crystals? Phys. Rev. Lett. 2012, 109, 195502.

(40) Ortiz, A. U.; Boutin, A.; Fuchs, A.; Coudert, F.-X. Investigating the Pressure-Induced Amorphization of Zeolitic Imidazolate Framework ZIF-8: Mechanical Instability due to Shear Mode Softening. J. Phys. Chem. Lett. 2013, 4, 1861-1865.

(41) We define $\phi$ as the fraction of porous volume over total volume; porous volume is what remains when excluding all spheres of radius $r_{i}$ $+r_{p}$, with $r_{i}$ the van der Waals radius of atom $i$ (we use $r_{\mathrm{Zn}}=1.39 \AA$, and $r_{\mathrm{C}}=r_{\mathrm{N}}=1.5 \AA$ ) and $r_{\mathrm{p}}=1.2 \AA$ the probe radius (smallest possible guest molecule).

(42) Both in the article and in the Supporting Information, unless otherwise stated, results and structures at $T=0 \mathrm{~K}$ refer to those obtained by DFT calculations.

\section{NOTE ADDED AFTER ASAP PUBLICATION}

This article was published ASAP on June 11, 2015, with an error in the abstract graphic. The corrected version was republished on June 12, 2015. 